Research Article

\title{
The Association between Adolescent's Weight Perception and Health Behaviors: Analysis of National Health and Nutrition Examination Survey Data, 2011-2014
}

\author{
Furong Xu $\mathbb{D}^{1},{ }^{1}$ Mary L. Greaney, ${ }^{2}$ Steven A. Cohen, ${ }^{2}$ Deborah Riebe, ${ }^{1}$ \\ and Geoffrey W. Greene $\mathbb{1}^{3}$ \\ ${ }^{1}$ Department of Kinesiology, University of Rhode Island, Independence Square II, Kingston, RI 02881, USA \\ ${ }^{2}$ Health Studies Program, University of Rhode Island, Independence Square II, Kingston, RI 02881, USA \\ ${ }^{3}$ Department of Nutrition and Food Sciences, University of Rhode Island, Fogarty Hall, Kingston, RI 02881, USA
}

Correspondence should be addressed to Furong Xu; fxu2007@uri.edu

Received 8 November 2017; Revised 21 March 2018; Accepted 2 April 2018; Published 23 April 2018

Academic Editor: David H. St-Pierre

Copyright (c) 2018 Furong Xu et al. This is an open access article distributed under the Creative Commons Attribution License, which permits unrestricted use, distribution, and reproduction in any medium, provided the original work is properly cited.

\begin{abstract}
The association between adolescents' weight perception and their physical activity (PA) and sedentary behaviors remains unclear. Therefore, these associations were explored using data from 2438 adolescents aged 12-19 years who participated in the National Health and Nutrition Examination 2011-2014 Survey. Respondents reported weight perception, and their weight perception accuracy was determined by examining whether the measured weight and perceived weight were concordant. Respondents also reported sedentary time (sitting time and screen time), PA, and intention to lose weight. Linear and logistic regression models were conducted to determine whether adolescents' PA, sedentary behaviors, and weight loss intention differed by weight perception and weight perception accuracy adjusted for demographic variables accounting for complex sampling. About onequarter (21.4\%) of the respondents had obesity. For respondents who perceived themselves as being overweight/fat, despite greater weight loss intention, males reported more sitting time $(512.7 \pm 16.3$ versus $474.1 \pm 10.2$ minutes/day, $p<0.05)$ and females reported less PA $(48.7 \pm 5.0$ versus $64.6 \pm 3.3$ minutes/day, $p<0.05)$ than respondents who perceived themselves as being normal weight. Similar patterns were observed for weight perception accuracy among individuals with obesity. Study results show that perceiving oneself as being overweight/fat regardless of accuracy was associated with more sedentary time for males or less PA for females despite higher weight loss intention.
\end{abstract}

\section{Introduction}

With $20.5 \%$ of adolescents aged $12-19$ years in the United States (US) classified as being overweight or having obesity, excess weight is epidemic and a pressing public health challenge [1]. Obesity-related healthcare costs are increasing and will likely burden the US healthcare system in the future [2]. Adolescents with obesity are more likely to have obesity as adults and as a result be at increased risk for chronic diseases [3, 4]. Efforts to reduce adolescent obesity have had limited success [1]. Inadequate physical activity (PA) contributes to obesity: adolescents who are obese are less physically active and more sedentary than their normal weight counterparts [5]. The median self-reported minutes of daily moderate-to-vigorous physical activity (MVPA) for adolescents with obesity is lower than that for their nonoverweight counterparts (24.8 minutes/day versus 37.1 minutes/day) and is well below the recommended 60 minutes/day of MVPA [5]. Conversely, adolescents with obesity spent on average 43 minutes more per day sitting than their nonoverweight counterparts [5].

Weight perception, the way in which individuals view their weight, influences individuals' weight concerns $[6,7]$. Although weight perception can be positive and may motivate weight control [8], negative weight perception can lead to extreme weight loss methods $[6,7]$. Thus, weight perception should be considered when developing programs to combat obesity. The literature examining the association 
between adolescents' weight perception and their weight control strategies, especially PA, is inconsistent [6, 9-13]. Some of the existing research studies indicate that adolescents who view themselves as being overweight regardless of their actual weight status are more likely to intend to lose weight but less likely to be physically active than a perception of being normal weight $[10,13]$, while other research studies have found that perceiving oneself as overweight may be associated with more PA than perceiving oneself as being normal weight [9]. Furthermore, existing research findings are also inconsistent when examining PA among adolescents who accurately perceive themselves as being overweight or obese $[6,11,13]$. One study found stronger weight loss intention and more PA participation [11], while other studies have found a lack of PA and more negative weight control behaviors such as extreme dieting $[6,13]$. Moreover, there is lack of research on adolescents' sitting time, in general. Given the emerging evidence of an association between sedentary behaviors and adolescent weight status [14], it is important to understand the association between weight perception and sedentary behaviors.

As rapid and diverse physical changes occur during adolescence and behavior patterns established in adolescence persist into adulthood [15], it is important to understand the relationships between weight perception and healthy behaviors to reduce obesity in adolescence [16, 17]. Yet, no identified studies that have examined weight perception and behaviors among US adolescents [6, 10-13] have assessed PA comprehensively or assessed sedentary sitting time in a nationally representative sample. Moreover, given the inconsistent evidence of the relationship between weight loss intention and healthy behaviors $[11,13]$, it is important to understand weight loss intention differences in relation to weight perception and weight perception accuracy. Accordingly, the purpose of this study was to examine the associations between adolescents' weight perception, weight perception accuracy, weight loss intention, PA, and sedentary behaviors, and how those potential associations may differ by sex. The primary hypothesis was that perceiving oneself as being overweight/fat would be associated with a greater intention to lose weight, more PA, and less sedentary time than those perceiving their weight as normal regardless of weight perception accuracy. The secondary hypothesis was that respondents who were accurately perceived themselves as having obesity would be more physically active, less sedentary, and have greater weight loss intentions than individuals with obesity who perceived their weight inaccurately.

\section{Methods}

The present study was a cross-sectional analysis of data from National Health and Nutrition Examination Survey (NHANES) 2011-2014. NHANES is a national health survey conducted by the Centers for Disease Control and Prevention (CDC) that includes questionnaires and an inperson examination conducted at a mobile examination center (MEC) [18]. The demographic questionnaire was completed by face-to-face interviews at the participants' homes by a parent or by the participants if 16 years of age or older. Participants aged 12-15 completed the PA questionnaire at an MEC while participants 16-19 years completed the questionnaire at home. Height and weight were measured at an MEC following standardized procedures [19].

2.1. Analytic Sample. The analytic sample was limited to adolescents who were 12-19 years old when they completed the NHANES examination $(n=2636)$. Of the available respondents, 95.8\% $(n=2526)$ had complete data for all examined variables. Individuals who were underweight $(n=88)$ based on calculated body mass index (BMI) were excluded due to the possibility that their weight status was related to psychological or physical pathology [20]. The final analytic sample included 2438 adolescents.

\subsection{Measures}

2.2.1. Weight Perception. Weight perception was determined by participants' response to the question "how you consider your weight," with the response options "overweight or fat," "too thin," and "about the right weight" [19]. Measured weight and height were used to calculate the BMI and to determine the weight status using CDC's sex-specific 2000 BMI-for-age growth reference for children and adolescents aged 2-20 years: underweight (BMI $<5$ th percentile), normal weight (BMI 5th to $<85$ th percentiles), overweight (BMI 85th to <95th percentiles), and obese (BMI $\geq 95$ th percentile) [21]. Weight perception accuracy (accurate versus inaccurate) was determined by comparing the perceived weight status and calculated the BMI weight status to determine if the two were concordant (accurate perceivers) or discordant (inaccurate perceivers).

2.2.2. Weight Loss Intention. Weight loss intention was assessed by a single item that asked participants what they were trying to do about their weight, with response options: "lose weight," "gain weight," "stay the same," or "do nothing." Weight loss intention was recoded as "trying to lose weight" (yes versus no) $[13,22,23]$. Only respondents aged 12-15 years $(n=1234)$ were asked this question [19].

2.2.3. Physical Activity (PA). PA was assessed using the Global Physical Activity Questionnaire (GPAQ) [19], a 16item instrument assessing frequency and duration of PA participation in three domains: (1) work-related PA which was assessed by 6 items; (2) travel-related PA which was assessed by 3 items; and (3) leisure time PA which was assessed by 6 items [24]. GPAQ scores for each of the 3 domains were summed and used to calculate MVPA time expressed by daily MVPA minutes or weekly metabolic equivalent (MET) minutes [25]. Participants were categorized as to whether they met the World Health Organization PA recommendation of at least 1680 MET-minutes per week for children or adolescent 12-17 years of age, and $600 \mathrm{MET}$ minutes per week for individuals 18-19 years of age [25]. 
2.2.4. Sedentary Time. Sedentary behaviors assessed in this study included sitting time and screen time. Sitting time was determined by participants' responses to a single item from the GPAQ that asked participants to report, in minutes, the time sitting or reclining on a typical day. Screen time was assessed by two items: (1) asked the participants to report the amount of time spent watching TV or videos and (2) asked the participants to report their daily computer use [25]. Responses (0-5+ hours per day) for both screen time items were summed and then used to determine whether participants met the American Academy of Pediatrics screen time guidelines ( $\leq 2$ hours/day) [26].

2.2.5. Covariates. Assessed demographic information included age, sex, race, parent education level (high school diploma or less versus some college or more), and the ratio of family income to poverty [19]. Responses for family income to poverty ratio (PIR) were used to create three household income categories: (1) PIR $\geq 3.5$ : at or above $350 \%$ of the poverty level; (2) $1.3 \leq$ PIR $<3.5$ : between $130 \%$ and $350 \%$ of the poverty level; and (3) PIR $<1.3$ : less than $130 \%$ of the poverty level [27].

2.3. Statistical Analysis. The MEC exam 2-year weights were used as the sample weight to conduct all analyses following the National Centre for Health Statistics recommendations [28]. Descriptive results were obtained for the sample and were presented as means \pm standard error for continuous variables and frequencies and proportions for categorical variables. To assess the bivariate associations between pairs of key predictor and outcome variables and to determine whether PA or sedentary time varied by weight perception and weight perception accuracy in males and females, $p$ values for continuous variables were obtained by performing PROC SURVEYREG (linear regression), and $p$ values for categorical variables were obtained by performing PROC SURVEYLOGISTIC (linear logistic regression), with STRATA, CLUSTER, and WEIGHT statements in these two types of models, adjusted for age, race, PIR, and parental education level. PROC SURVEYFREQ (CHISQ, based on the Rao-Scott chi-square test with an adjusted $F$ statistic) was also conducted to compare the difference in proportion when no adjustment was required.

Lastly, sensitivity analyses were conducted because of the difference in time spent on work-related PA between adolescents aged $16-19$ and $12-15$ years $(42.7 \pm 9.9$ versus $11.0 \pm$ 3.1 minutes/day, $p=0.005$ ) [29]. For this analysis, workrelated PA was excluded from the total PA, and no meaningful differences in the results were found. Statistical significance was set at $p<0.05$ for all analyses. All statistical analyses were conducted using SAS version 9.4 (SAS Institute Inc., Cary, NC).

\section{Results}

Of the 2438 adolescents in the study sample, approximately half (49.2\%) were girls, $45.4 \%$ were racial/ethnic minorities, $42.9 \%$ had a parent who had a high school degree or less, and
$33.7 \%$ of the participants lived below $130 \%$ of the poverty line (PIR <1.3). About one-fifth of boys $(20.8 \%)$ and girls (21.9\%) were classified as having obesity (See Table 1 for sample characteristics stratified by sex and weight status).

Sex-specific analysis revealed that PA, sitting time, and screen time differed by weight perception categories (thin, normal, and overweight/fat). For boys, individuals who considered themselves to be overweight/fat were more likely to intend to lose weight ( $87.5 \%$ versus $22.4 \%$ ) than boys who perceived themselves as being normal weight; however, they were less likely to meet PA recommendations (64.0\% versus $76.3 \%)$, spend more time sitting $(512.7 \pm 16.3$ versus $474.1 \pm 10.2$ minutes/day), and had greater screen time ( $4.4 \pm 0.2$ versus $3.6 \pm 0.1$ hours/day) (Table 2$)$. Among girls, individuals who perceived themselves as being overweight/fat were more likely to intend to lose weight (91.6\% versus $29.4 \%$ ) but reported less PA time $(48.7 \pm 5.0$ versus $64.6 \pm 3.3$ minutes/day) than those who perceived themselves as normal weight (Table 2).

Comparisons of PA, sedentary time, and weight loss intentions among accurate weight perceivers revealed similar patterns as above (Table 3). In general, normal weight boys and girls perceived their weight accurately (87\% and $87 \%$, resp.) as did boys and girls who were obese (66\% and $76 \%$, resp.) but a smaller percentage of overweight boys and girls were accurate weight perceivers (21\% and $49 \%$, resp.). Boys with accurate weight perceptions who were obese were more likely to intend to lose weight than normal weight accurate perceivers (90.3\% versus 12.8\%); however, they were less likely to meet the PA (62.4\% versus $77.4 \%)$ and screen time $(24.7 \%$ versus $36.4 \%$ ) recommendations and had more daily sitting time $(535.6 \pm 21.3$ versus $468.1 \pm 9.6$ minutes/day) than normal weight boys. Among girls who perceived their weight status accurately, respondents with obesity were more likely to intend to lose weight ( $89.5 \%$ versus $19.8 \%$ ) but were less likely to meet screen time recommendations ( $29.7 \%$ versus $42.9 \%)$ and were more physically inactive $(41.0 \pm 4.1$ minutes/day versus $64.2 \pm 3.5$ minutes/day) than normal weight girls (Table 3).

Sex-specific analysis by weight perception accuracy among respondents classified as having obesity revealed differences in PA, sedentary time, and weight loss intentions by weight perception accuracy. Among boys who were obese, weight perception accuracy was associated with increased sitting time $(535.6 \pm 21.3$ versus $497.8 \pm 31.9$ minutes/day, $p=0.007)$, being less likely to meet screen time recommendations $(24.7 \%$ versus $34.0 \%, p=0.036)$, and having a greater intention to lose weight (90.3\% versus $56.4 \%$, $p=0.004$ ) than inaccurate perceivers (Table 3). Among girls with obesity, accurate weight perceivers were less physically active ( $41.0 \pm 4.1$ versus $61.7 \pm 12.2$ minutes/day, $p=0.005)$ and have a greater intention to lose weight $(89.5 \%$ versus $66.9 \%, p=0.046)$ than those who inaccurately perceived their weight (Table 3).

\section{Discussion}

The current study examined adolescents' PA and sedentary behaviors by weight perception and weight perception 


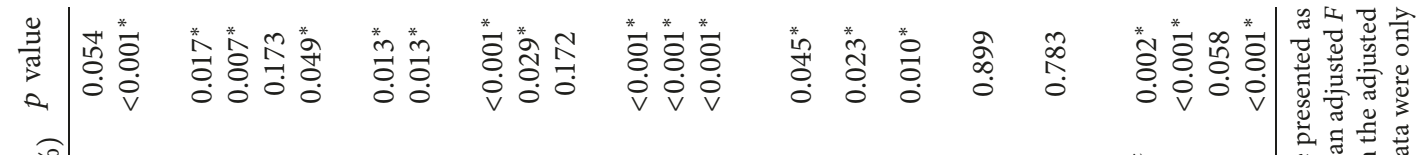

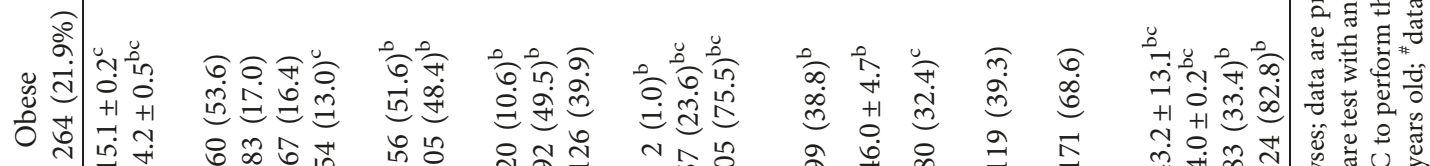

II

$\begin{array}{rlllllllll} & & & & \\ 0\end{array}$

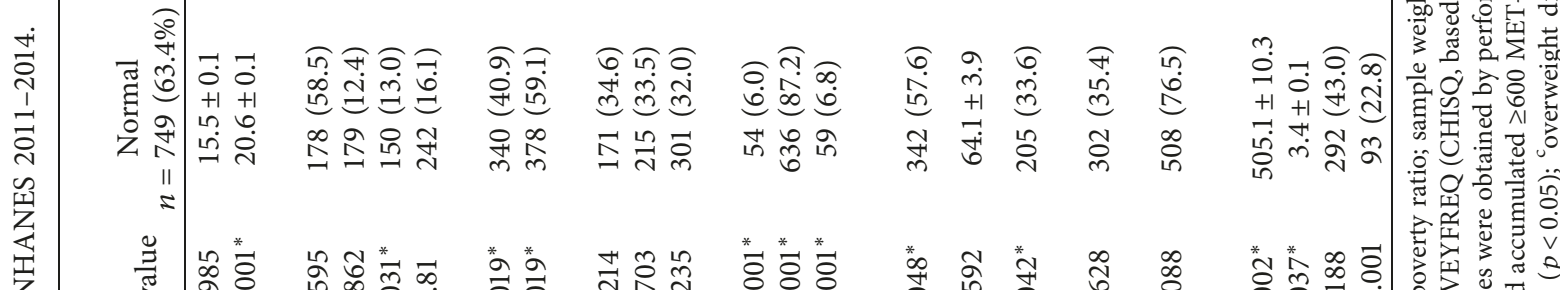

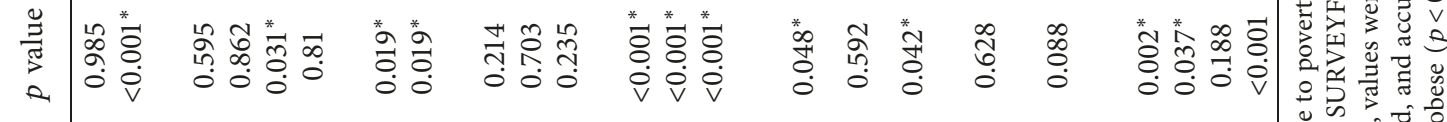

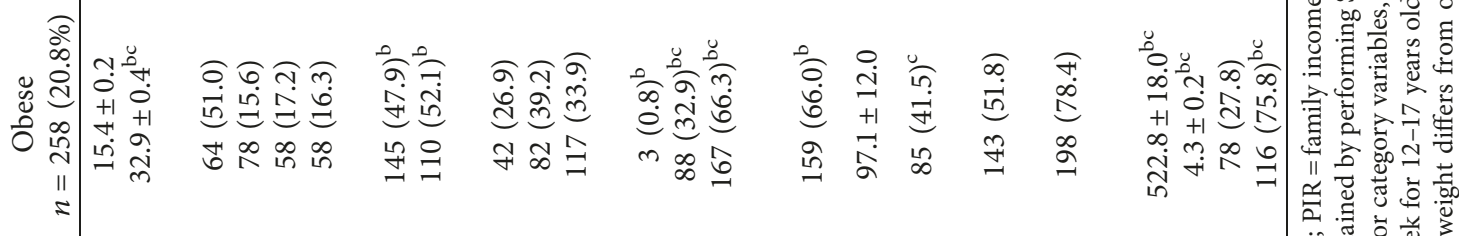

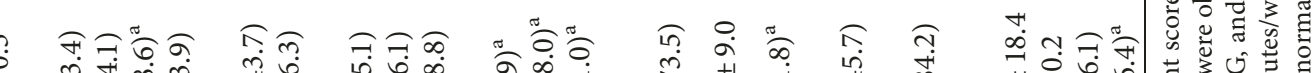

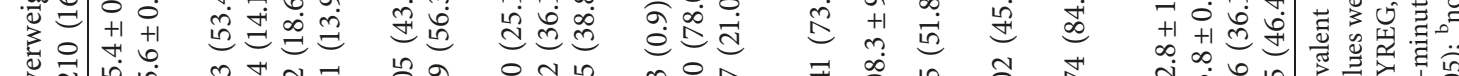

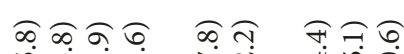

ชุำ

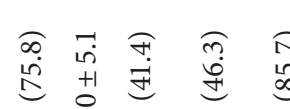

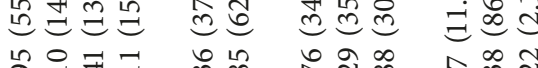

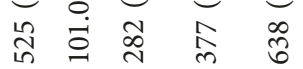

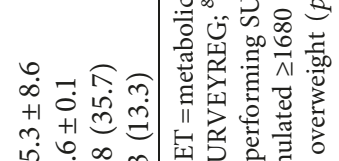

乙罂战完

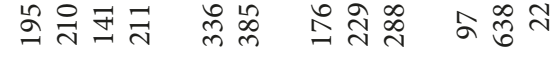

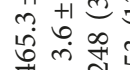

空

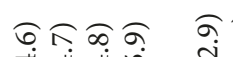

भaิ

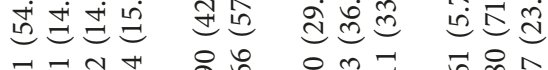

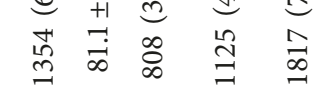

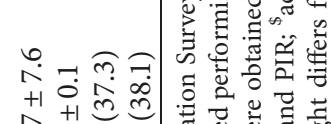


TABLE 2: Weight perception and health behaviors in adolescents aged 12-19 years, NHANES 2011-2014.

\begin{tabular}{|c|c|c|c|c|c|}
\hline & Total & Thin & Normal & Overweight/fat & Overall $p$ value \\
\hline \multicolumn{6}{|l|}{ Boys } \\
\hline $12-19$ years & $n=1225$ & $n=103(7.3 \%)$ & $n=886(74.1 \%)$ & $n=236(18.6 \%)$ & \\
\hline \multicolumn{6}{|l|}{ Physical activity } \\
\hline Meet physical activity recommendation ${ }^{\$}, n(\%)$ & $825(73.4)$ & $70(67.2)$ & $616(76.3)$ & $139(64.0)^{\mathrm{a}}$ & $<0.001^{*}$ \\
\hline Physical activity (minutes/day) & $101.4 \pm 4.6$ & $117.3 \pm 19.1$ & $101.2 \pm 4.4$ & $96.3 \pm 10.8$ & 0.187 \\
\hline \multicolumn{6}{|l|}{ Sedentary behaviors } \\
\hline Sitting time (minutes/day) & $478.5 \pm 8.0$ & $436.0 \pm 20.1$ & $474.1 \pm 10.2$ & $512.7 \pm 16.3^{\mathrm{ab}}$ & $<0.001^{*}$ \\
\hline Screen time (hours/day) & $3.7 \pm 0.1$ & $3.6 \pm 0.3$ & $3.6 \pm 0.1$ & $4.4 \pm 0.2^{\mathrm{ab}}$ & $0.004^{*}$ \\
\hline Screen time $\leq 2$ hours/day, $n(\%)$ & $402(34.1)$ & $33(31.4)$ & $299(36.4)$ & $70(26.0)^{\mathrm{a}}$ & $0.017^{*}$ \\
\hline $12-15$ years & $n=626$ & $n=49(6.2 \%)$ & $n=465(78.1 \%)$ & $n=112(15.8 \%)$ & \\
\hline Weight loss intention, $n(\%)^{\#}$ & $224(32.0)$ & $3(12.1)$ & $119(22.4)$ & $102(87.5)^{\mathrm{ab}}$ & $<0.001$ \\
\hline \multicolumn{6}{|l|}{ Girls } \\
\hline $12-19$ years & $n=1213$ & $n=58(4.1 \%)$ & $n=794(67.9 \%)$ & $n=361(28 \%)$ & \\
\hline \multicolumn{6}{|l|}{ Physical activity } \\
\hline Meet physical activity recommendation $\$, n(\%)$ & $529(51.8)$ & $22(46.1)$ & $369(56.6)$ & $138(41.0)^{\mathrm{a}}$ & $0.008^{*}$ \\
\hline Physical activity (minutes/day) & $60.0 \pm 2.6$ & $61.1 \pm 11.4$ & $64.6 \pm 3.3$ & $48.7 \pm 5.0^{\mathrm{a}}$ & $0.021^{*}$ \\
\hline \multicolumn{6}{|l|}{ Sedentary behaviors } \\
\hline Sitting time (minutes/day) & $507.3 \pm 9.0$ & $489.8 \pm 23.4$ & $508.6 \pm 10.6$ & $506.7 \pm 15.1$ & 0.377 \\
\hline Screen time (hours/day) & $3.5 \pm 0.1$ & $3.1 \pm 0.4$ & $3.5 \pm 0.1$ & $3.8 \pm 0.2$ & 0.135 \\
\hline Screen time $\leq 2$ hours/day, $n(\%)$ & $442(40.5)$ & $23(44.7)$ & $311(43.2)$ & $108(33.3)$ & 0.153 \\
\hline $12-15$ years & $n=608$ & $n=32(4.7 \%)$ & $n=423(69.9 \%)$ & $n=153(25.3 \%)$ & \\
\hline Weight loss intention, $n(\%)^{\#}$ & $285(44.3)$ & $3(9.8)$ & $143(29.4)^{\mathrm{c}}$ & $139(91.6)^{\mathrm{ab}}$ & $<0.001$ \\
\hline
\end{tabular}

Note. NHANES = National Health and Nutrition Examination Survey; MET = metabolic equivalent scores; sample weight to conduct all the analyses; data are presented as mean \pm SE or otherwise specified; the other $p$ values for continuous variable were obtained by performing SURVEYREG, and $p$ values for category variables were obtained by performing SURVEYLOGISTIC to perform the adjusted analyses, adjusted for age, race, parental education level, and family income to poverty ratio; ${ }^{\$}$ accumulated $\geq 1680$ MET-minutes/week for 12-17 years old, and accumulated $\geq 600$ MET-minutes/week for 18-19 years old; \#data were only available for participants aged 12-15 years; ${ }^{a}$ normal differs from overweight $(p<0.05)$; ${ }^{b}$ thin differs from overweight $(p<0.05)$; ${ }^{c}$ thin differs from normal weight $(p<0.05)$.

accuracy in a nationally representative sample. We found that respondents, regardless of their weight perception accuracy, who perceived themselves as being overweight/fat were more sedentary or less physically active. This finding provides valuable insight into obesity intervention programs designed to help adolescents overcome barriers to PA participation and reduce sedentary time.

In total, $34 \%$ of boys and $24 \%$ of girls who were classified as obese perceived their weight status inaccurately, which is similar to the proportion ( $40 \%$ boys versus $23 \%$ girls) from the Youth Risk Behavior Surveillance System, 1999-2007 [11]. Results of the current study confirm prior research that has shown that both boys and girls who perceive their weight as overweight/fat are less physically active than boys and girls who perceive their weight as being normal, regardless of weight perception accuracy $[11,13]$. This suggests that weight perception may be a determinant of PA participation for boys and girls. Moreover, study findings indicate that accurately perceiving oneself as being overweight/fat does not necessarily motivate individuals to engage in more PA, as there was no difference in PA among boys with obesity by weight perception accuracy. However, female respondents who accurately perceived themselves as being obese had less average daily PA time than inaccurate weight perceivers. This finding differs from that of Fan and Jin's who found that boys with accurate weight perception of overweight were less physically active, whereas there was no PA difference in girls [13]. These different findings could be due to sample differences as their samples were older (9-12th grade students), difference in actual weight categories (overweight and obese were combined), and differences in PA assessment $[13,19]$. The current study assessed three types of PA (work, travel, and leisure) and is the first, to our knowledge, to explore weight perception and PA using multiple PA domains, which is important because adolescents' PA is not necessarily limited to recreational PA [30-32]. Around $40 \%$ of respondents in our study reported work- or travel-related PA. Thus, the current study adds to the literature as it provides evidence that PA occurs in different domains and suggests that adolescent obesity prevention program should be tailored to PA in different domains.

A novel study finding is that boys and not girls, who perceived themselves as being overweight/fat had greater sitting time in general and more screen time than boys who perceived themselves as being thin or normal weight. This finding of a specific difference in sitting time and screen time may help future health promotion efforts addressing sedentary behaviors. Moreover, when taking weight perception accuracy into consideration, both boys and girls were accurately perceived themselves as having obesity reported more screen time than those who accurately perceived themselves as being normal weight, which was consistent with that of Fan and Jin [13]. Since sitting time and screen time may be associated with an increased risk for obesity $[33,34]$, it is important to address sedentary behaviors via appropriate and effective guidance and support to reduce obesity among adolescents.

Results of this study also indicate that adolescents who viewed themselves as overweight/fat were more likely to express weight loss intentions than those who perceived 


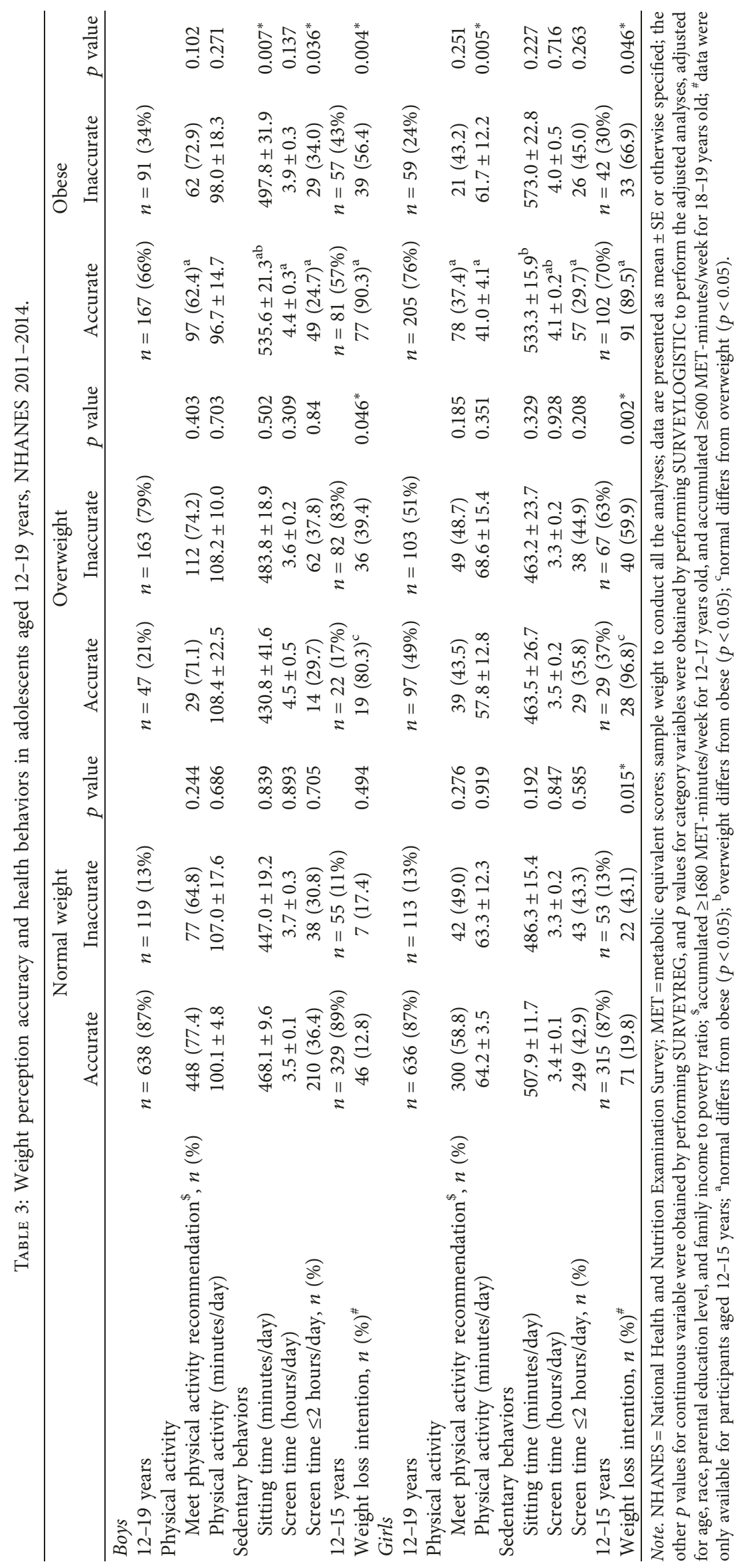


themselves as being normal weight, which is consistent with previous research $[13,26]$, although weight loss intention was not associated with greater PA. Moreover, respondents with obesity who accurately perceived their weight status were more likely to express weight loss intention than inaccurate weight perceivers, which aligns with the findings of Fan and Jin [13]. This finding along with the study findings on PA and sedentary time indicates that adolescents who are aware of their weight and intend to lose weight may need appropriate and effective guidance and support to overcome barriers to PA and make desirable behavior changes. It is also important to recognize that weight loss intention can be associated with potentially dangerous weight control practices. Dieting is a common weight control practice among adolescents with obesity, although dieting is often counterproductive leading to long-term weight gain $[35,36]$. However, assessing dieting behavior is beyond the scope of the present study. Increasing MVPA time and decreasing screen time are positive weight control strategies that contribute to a healthy lifestyle pattern [37]. Study findings suggest that weight perception is associated with weight loss intention; however, there is a need to address barriers to PA participation among adolescents perceiving themselves as overweight/fat to help them transform weight loss intentions into effective health promoting behaviors.

Study findings should be interpreted with caution due to the study's cross-sectional design, which does not allow for causality to be determined. In addition, PA, sitting time, and screen time were assessed by self-reported measures, which may induce bias. Despite these limitations, PA was assessed by a validated instrument that been widely used internationally and in similar studies of adolescent obesity $[24,38,39]$. Also, due to the secondary nature of this data analysis, we were limited to the questions already asked in the survey and not able to conduct more in-depth analyses. For example, the available response to the question assessing weight perception responses did not separate overweight and obese, and weight loss intention data were only available for participants aged $12-15$ years.

Strengths of this study include the use of the recent nationally representative sample to investigate and the use of measured height and weight to calculate the BMI and to determine weight perception accuracy. In addition, the PA measure assessed multiple domains of PA, and no similar studies have included work and travel PA measures or sitting time. Lastly, a sensitivity analysis was conducted, which confirmed primary findings when work-related PA was excluded from the total PA time.

\section{Conclusion}

A perception of being overweight/fat in adolescents was associated with greater weight loss intention but more sedentary time for boys or less PA time for girls than a perception of being normal weight regardless of weight perception accuracy. Weight perception accuracy was not associated with more PA or less sedentary time among individuals with obesity despite their greater intention to lose weight. This finding is important for obesity prevention/intervention programs or future health promotion efforts aimed to help adolescents improve PA participation and reduce sedentary time.

\section{Disclosure}

The content of this paper is the responsibility of the authors and does not necessarily represent the official views of Centers for Disease Control and Prevention.

\section{Conflicts of Interest}

The authors declare that there are no conflicts of interest regarding this paper.

\section{References}

[1] C. L. Ogden, M. D. Carroll, H. G. Lawman et al., "Trends in obesity prevalence among children and adolescents in the United States, 1988-1994 through 2013-2014," JAMA, vol. 315, no. 21 , pp. 2292-2299, 2016.

[2] G. Wang and W. H. Dietz, "Economic burden of obesity in youths aged 6 to 17 years: 1979-1999," Pediatrics, vol. 109, no. 5 , p. e81, 2002.

[3] N. S. The, C. Suchindran, K. E. North, B. M. Popkin, and P. Gordon-Larsen, "The association of adolescent obesity with risk of severe obesity in adulthood," JAMA, vol. 304, no. 18, pp. 2042-2047, 2010.

[4] D. S. Freedman, W. H. Dietz, S. R. Srinivasan, and G. S. Berenson, "The relation of overweight to cardiovascular risk factors among children and adolescents: the Bogalusa heart study," Pediatrics, vol. 103, no. 6, pp. 1175-1182, 1999.

[5] V. Carson, A. E. Staiano, and P. T. Katzmarzyk, "Physical activity, screen time, and sitting among US adolescents," Pediatric Exercise Science, vol. 27, no. 1, pp. 151-159, 2015.

[6] W. M. Felts, A. V. Parrillo, T. Chenier, and P. Dunn, “Adolescents' perceptions of relative weight and self-reported weight-loss activities: analysis of 1990 YRBS national data," Journal of Adolescent Health, vol. 18, no. 1, pp. 20-26, 1996.

[7] D. Voelker, J. Reel, and C. Greenleaf, "Weight status and body image perceptions in adolescents: current perspectives," Adolescent Health, Medicine and Therapeutics, vol. 6, pp. 149-158, 2015.

[8] T. Baranowski, K. Cullen, T. Nicklas, D. Thompson, and J. Baranowski, "Are current health behavioral change models helpful in guiding prevention of weight gain efforts?," Obesity Research, vol. 11, pp. 23S-43S, 2003.

[9] S. L. Wong and S. T. Leatherdale, "Association between sedentary behavior, physical activity, and obesity: inactivity among active kids," Prevention of Chronic Disease, vol. 6, no. 1, p. A26, 2009.

[10] J. Yost, B. Krainovich-Miller, W. Budin, and R. Norman, "Assessing weight perception accuracy to promote weight loss among U.S. female adolescents: a secondary analysis," BMC Public Health, vol. 10, p. 465, 2010.

[11] N. M. Edwards, S. Pettingell, and I. W. Borowsky, "Where perception meets reality: self-perception of weight in overweight adolescents," Pediatrics, vol. 125, no. 3, pp. e452-e458, 2010.

[12] A. E. Chung, E. M. Perrin, and A. C. Skinner, "Accuracy of child and adolescent weight perceptions and their relationships to dieting and exercise behaviors: NHANES," Academic Pediatrics, vol. 13, no. 4, pp. 371-378, 2013. 
[13] M. Fan and Y. Jin, "The effects of weight perception on adolescents' weight-loss intentions and behaviors: evidence from the youth risk behavior surveillance survey," International Journal of Environmental Research and Public Health, vol. 12, no. 11, pp. 14640-14668, 2015.

[14] J. A. Mitchell, R. R. Pate, M. W. Beets, and P. R. Nader, "Time spent in sedentary behavior and changes in childhood BMI: a longitudinal study from ages 9 to 15 years," International Journal of Obesity, vol. 37, pp. 54-60, 2013.

[15] J. Calzo, K. R. Sonneville, J. Haines, E. A. Blood, A. E. Field, and S. B. Austin, "The development of associations among body mass index, body dissatisfaction, and weight and shape concern in adolescent boys and girls," Journal of Adolescent Health, vol. 51, no. 5, pp. 517-523, 2012.

[16] F. Kuchler and J. N. Variyam, "Mistakes were made: misperception as a barrier to reducing overweight," International Journal of Obesity, vol. 27, pp. 856-861, 2003.

[17] C. O. Gregory, H. M. Blanck, C. Gillespie, L. M. Maynard, and M. K. Serdula, "Health perceptions and demographic characteristics associated with underassessment of body weight," Obesity, vol. 16, pp. 979-986, 2008.

[18] C. L. Johnson, S. M. Dohrmann, V. L. Burt, and L. K. Mohadjer, "National health and nutrition examination survey: sample design, 2011-2014," Vital and Health Statistics, Series 2, vol. 162, pp. 1-33, 2014.

[19] Centers for Disease Control and Prevention, National Health and Nutrition Examination Survey, July 2017, https://wwwn. cdc.gov/nchs/nhanes.

[20] K. Nadal, The Sage Encyclopedia of Psychology and Gender, Sage, Thousand Oaks, CA, USA, 2017.

[21] C. L. Ogden, R. J. Kuczmarski, K. M. Flegal et al., "Centers for disease control and prevention 2000 growth charts for the united states: improvements to the 1977 national center for health statistics version," Pediatrics, vol. 109, no. 1, pp. 45-60, 2002.

[22] J. Fredrickson, P. Kremer, B. Swinburn, A. de Silva, and M. McCabe, "Weight perception in overweight adolescents: associations with body change intentions, diet and physical activity," Journal of Health Psychology, vol. 20, no. 6, pp. 774-784, 2015.

[23] H. Bittner Fagan, J. Diamond, R. Myers, and J. M. Gill, "Perception, intention, and action in adolescent obesity," Journal of the American Board of Family Medicine, vol. 21, no. 6 , pp. 555-561, 2008.

[24] T. Armstrong and F. Bull, "Development of the World Health Organization Global Physical Activity Questionnaire (GPAQ)," Journal of Public Health, vol. 14, no. 2, pp. 66-70, 2006.

[25] World Health Organization, Global Physical Activity Questionnaire (GPAQ) Analysis Guide, August 2017, https://www. who.int/chp/steps/resources/GPAQ_Analysis_Guide.pdf.

[26] American Academy of Pediatrics, "Committee on public education. American academy of pediatrics: children, adolescents, and television," Pediatrics, vol. 107, pp. 423-426, 2001.

[27] C. L. Ogden, M. M. Lamb, M. D. Carroll, and K. M. Flegal, "Obesity and socioeconomic status in children and adolescents: United States, 2005-2008," NCHS Data Brief, vol. 51, pp. 1-8, 2010.

[28] CDC National Center for Health Statistics, Specifying Weighting Parameters, August 2017, https://www.cdc.gov/nchs/tutorials/nhanes/ surveydesign/weighting/intro.htm.

[29] L. Thabane, L. Mbuagbaw, S. Zhang et al., "A tutorial on sensitivity analyses in clinical trials: the what, why, when and how," BMC Medical Research Methodology, vol. 13, p. 92, 2013.

[30] B. I. Saksvig, D. J. Catellier, K. Pfeiffer et al., "Travel by walking before and after school increases physical activity among adolescent girls," Archives of Pediatrics and Adolescent Medicine, vol. 161, no. 2, pp. 153-158, 2007.

[31] K. K. Davison, J. L. Werder, and C. T. Lawson, "Children's active commuting to school: current knowledge and future directions," Preventing Chronic Disease, vol. 5, no. 3, p. A100, 2008.

[32] C. Hirschman and I. Voloshin, "The structure of teenage employment: social background and the jobs held by high school seniors," Research in Social Stratification and Mobility, vol. 25, no. 3, pp. 189-203, 2007.

[33] M. S. Tremblay, A. G. LeBlanc, M. E. Kho et al., "Systematic review of sedentary behaviour and health indicators in schoolaged children and youth," International Journal of Behavioral Nutrition and Physical Activity, vol. 8, p. 98, 2011.

[34] N. Pearson and S. J. H. Biddle, "Sedentary behaviour and dietary intake in children, adolescents and adults: a systematic review," American Journal of Preventive Medicine, vol. 41, no. 2, pp. 178-188, 2011.

[35] K. Boutelle, D. Neumark-Sztainer, M. Story, and M. Resnick, "Weight control behaviors among obese, overweight, and non-overweight adolescents," Journal of Pediatric Psychology, vol. 27, no. 6, pp. 531-540, 2002.

[36] D. Neumark-Sztainer, M. Wall, J. Guo, M. Story, J. Haines, and M. Eisenberg, "Obesity, disordered eating, and eating disorders in a longitudinal study of adolescents: how do dieters fare 5 years later?," Journal of the American Dietetic Association, vol. 106, no. 4, pp. 559-568, 2006.

[37] A. Must and D. J. Tybor, "Physical activity and sedentary behavior: a review of longitudinal studies of weight and adiposity in youth," International Journal of Obesity, vol. 29, no. S2, pp. S84-S96, 2005.

[38] S. Kaviraj, A. Sinha, N. Chakraborty, H. Roy, R. Majumdar, and M. M. Mondal, "Physical activity status and body image perception of adolescent females in a slum in Kolkata, India," IOSR-Journal of Dental and Medical Sciences, vol. 10, no. 1, pp. 11-14, 2013.

[39] A. Al-Zalabani, N. A. Al-Hamdan, and A. A. Saeed, "The prevalence of physical activity and its socioeconomic correlates in Kingdom of Saudi Arabia: a cross-sectional population-based national survey," Journal of Taibah University Medical Sciences, vol. 10, no. 2, pp. 2018-2215, 2015. 


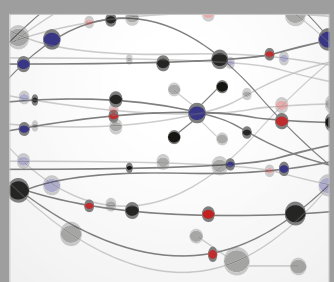

The Scientific World Journal
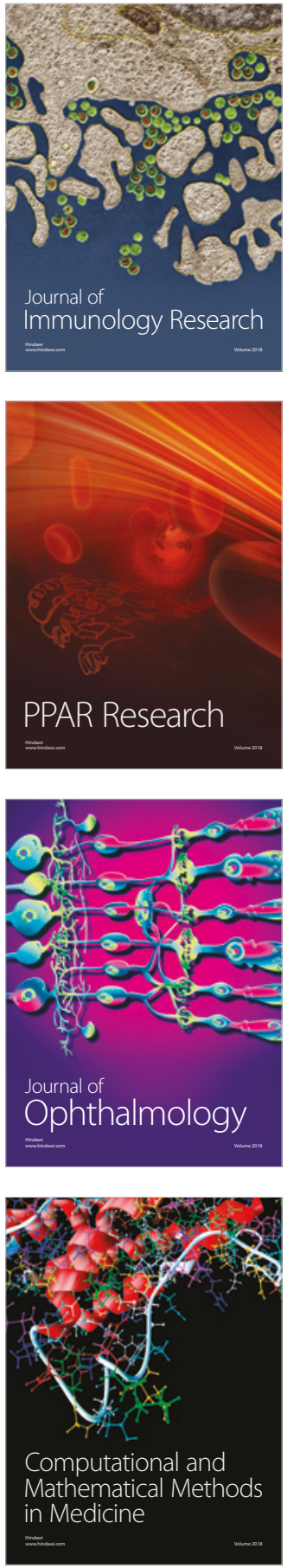

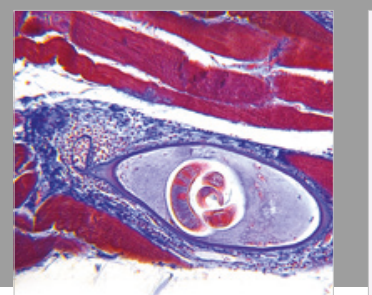

Gastroenterology Research and Practice

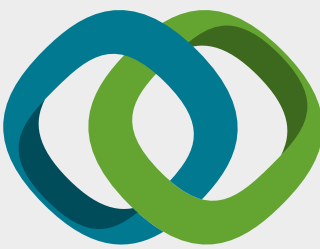

\section{Hindawi}

Submit your manuscripts at

www.hindawi.com
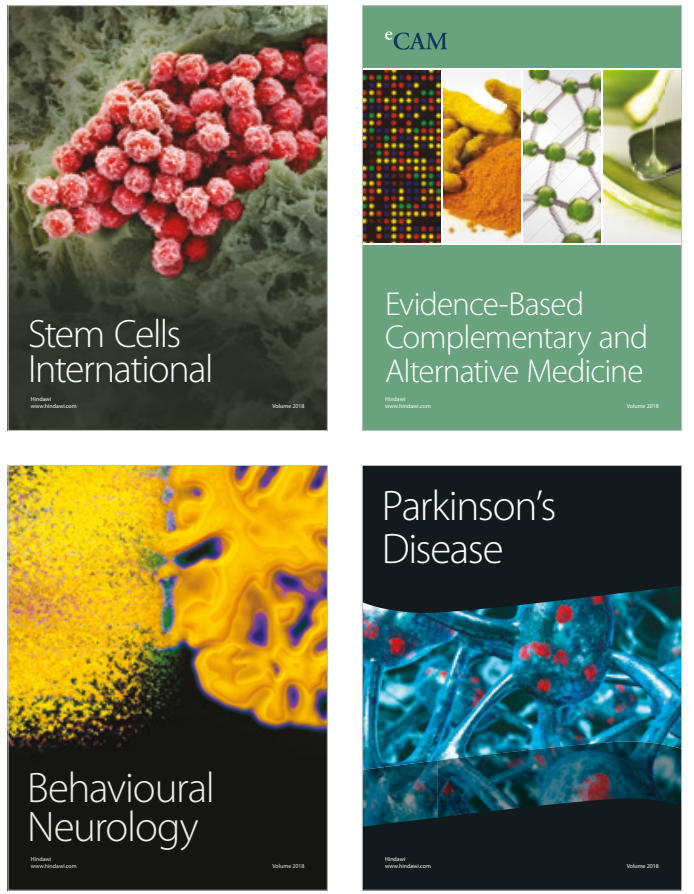

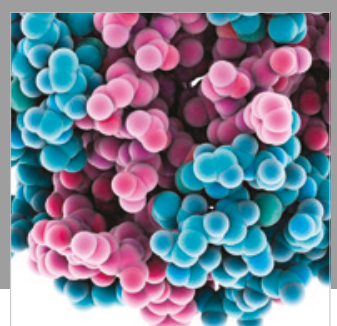

ournal of

Diabetes Research

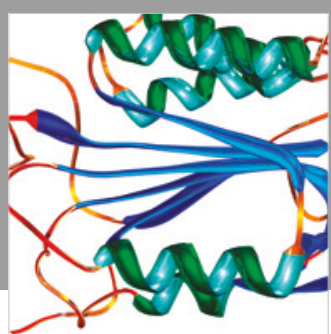

Disease Markers
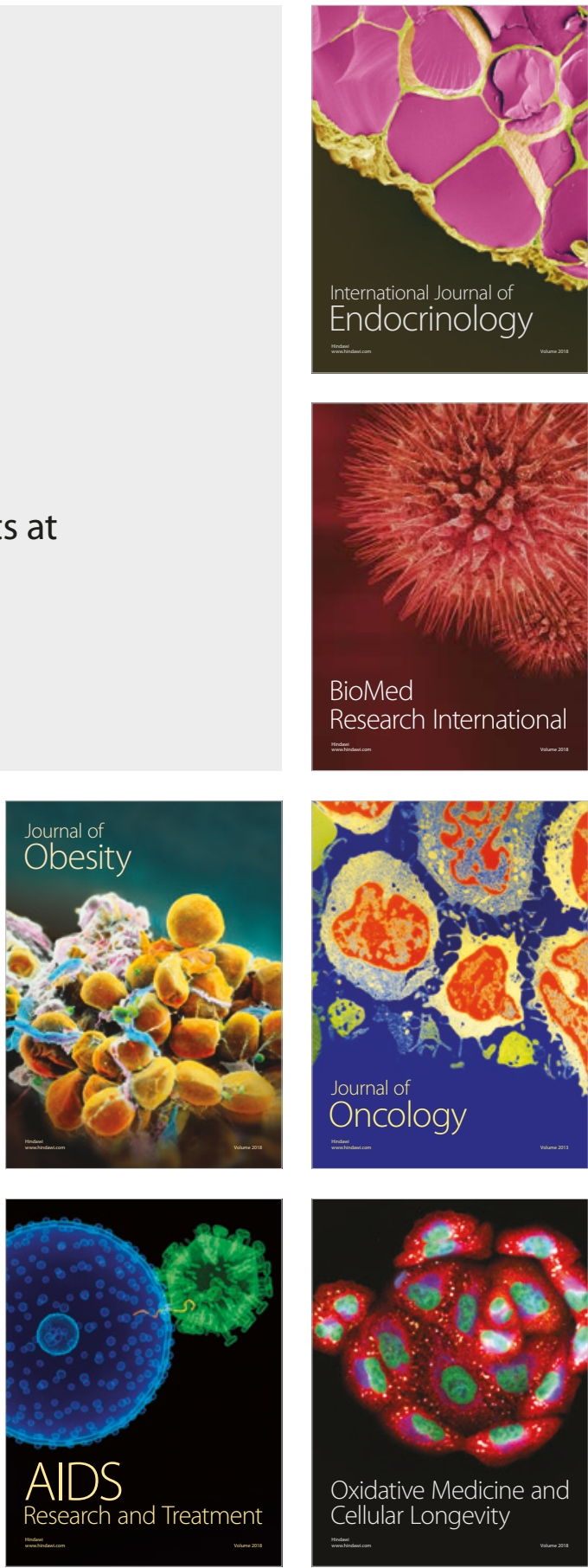